\title{
Gastric cancer diagnosed in a patient with crescentic glomerulonephritis
}

\author{
Tugba Yavuzsen ${ }^{1}$, Ilhan Oztop ${ }^{1}$, Ugur Yilmaz $^{1}$, Caner Cavdar $^{2}$, Aykut Sifil $^{2}$, Sulen Sarioglu ${ }^{3}$, \\ and Mehmet Alakavuklar ${ }^{1}$ \\ ${ }^{1}$ University of Dokuz Eylul, Institute of Oncology, Balcova, 35340 Izmir, Turkey \\ ${ }^{2}$ University of Dokuz Eylul, Faculty of Medicine, Department of Internal Medicine, Divison of Nephrology, Izmir, Turkey \\ ${ }^{3}$ University of Dokuz Eylul, Faculty of Medicine, Department of Pathology, Izmir, Turkey
}

\begin{abstract}
Malignant neoplasms are rarely associated with glomerular changes. The glomerular lesion seen most often in patients with malignancies is membranous glomerulonephritis. We report here a case of gastric cancer associated with rapidly progressive glomerulonephritis (RPGN). A 57-year-old man was admitted to our hospital with acute renal failure. Renal biopsy was performed, and the diagnosis was RPGN. Steroid treatment was instituted, but was complicated by hematemesis, and an early-stage gastric cancer was found after endoscopic examinations. Gastric biopsy revealed gastric adenocarcinoma. The patient's renal function improved with corticosteroid treatment and hemodialysis, and total gastrectomy was performed. The improvement in renal function persisted after the removal of the gastric cancer, and the RPGN and cancer have been in remission for 3 years.
\end{abstract}

Key words Gastric cancer · Rapidly progressive glomerulonephritis

\section{Introduction}

Malignant neoplasms are rarely associated with glomerular changes, and these changes are most often considered to be paraneoplastic events. Such changes have been reported with nearly all types of cancers, including adenocarcinoma of the gastrointestinal tract. The glomerular disorder most often encountered in patients with malignancies is membranous glomerulonephritis (MGN). Other glomerular pathologies, such as minimal change disease, mesangio-capillary glomerulonephritis, IgA nephropathy, crescentic nephritis, and amyloidosis have also been reported in patients with neoplastic diseases [1-5]. Here we report a patient who presented

Offprint requests to: T. Yavuzsen

Received: April 9, 2003 / Accepted: August 19, 2003 with rapidly progressive glomerulonephritis and simultaneously had a diagnosis of gastric cancer.

\section{Case report}

A 57-year old man was admitted to our hospital with complaints of fever, fatigue, and oliguria. History revealed a recent upper respiratory infection, and he had been taking oral penicillin for 7 days. There was no history of significant disorders such as arthritis, diabetes, skin changes, vasculopathy, neuropathy, and others. Physical examination was normal; no edema was observed and blood pressure was normal, being 130/ $85 \mathrm{mmHg}$ on admission and normal thereafter. The blood count findings were within normal limits (hemoglobin $[\mathrm{Hb}], 13.1 \mathrm{~g} / \mathrm{dl}$; WBC, $7 \times 10^{9} / 1$; platelets, $106 \times$ $\left.10^{9} / 1\right)$. However, blood chemistry revealed abnormal renal function (blood urea nitrogen [BUN], $69 \mathrm{mg} / \mathrm{dl}$ [upper normal limit; UNL], $20 \mathrm{mg} / \mathrm{dl}$ ); creatinine, $7.2 \mathrm{mg} / \mathrm{dl}(\mathrm{UNL}, 1.4 \mathrm{mg} / \mathrm{dl}$ ); and $\mathrm{K}, 4.8 \mathrm{mMol} / \mathrm{l}$ (UNL, $5.5 \mathrm{~m} \mathrm{Mol} / \mathrm{l}$ ), but transaminases, blood sugar, and coagulation test results were within normal limits. On examination of spot urine, mild proteinuria (approximately $500 \mathrm{mg} / \mathrm{dl}$ ) and mild hematuria with waxy RBC casts were found. Blood gases and electrocardiography were normal. Abdominal ultrasonography on admission showed an increase of renal dimensions (right kidney measured $123 \times 60 \mathrm{~mm}$; left kidney, $140 \times 76 \mathrm{~mm})$ and increased parenchymal thickness $(10 \mathrm{~mm}$ in right kidney, $18 \mathrm{~mm}$ in left kidney). The echogenicity of both kidneys was increased and was considered to be grade II. Autoantibodies, such as anti-glomerular basement membrane (GBM) antibody, perinuclear antineutrophil cytoplasmic autoantibodies (p-ANCA), cytoplasmic antineutrophil cytoplasmic autoantibodies (c-ANCA), antinuelear antibodies (ANA), and anti-double strand DNA (anti-[Ds]DNA) were negative, and cryoglobulin, direct and indirect Coombs tests, and markers of 


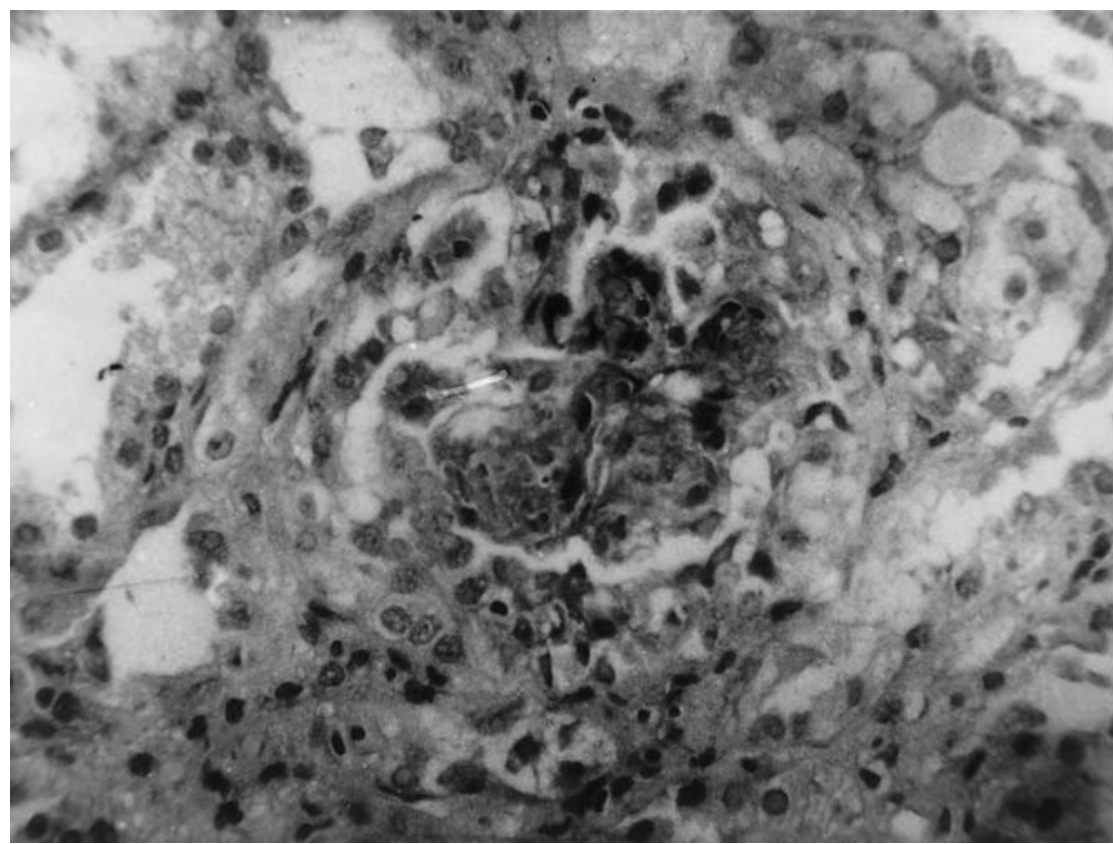

Fig. 1. Glomerulus with necrosis and crescent formation. $\mathrm{H} \& \mathrm{E}, \times 40$ hepatitis $\mathrm{B}$ and $\mathrm{C}$ were negative. Serum complement 3 (C3) and 4 (C4) levels were high (C3, 0.64 mg/dl; UNL, $0.1 \mathrm{mg} / \mathrm{dl}$, and C4, $0.18 \mathrm{mg} / \mathrm{dl}$; UNL, $0.5 \mathrm{mg} / \mathrm{dl}$ ). Anticardiolipin antibody could not be analyzed.

Hemodialysis was performed as an emergency procedure for acute renal failure on the day of his admission to the hospital. A renal biopsy was performed 2 days later, and histopathologic examination was in favor of a diagnosis of pauci-immune rapidly progressive glomerulonephritis (RPGN; crescentic glomerulonephritis) with membranous and mesangial deposition of C3, fibrin, and albumin (Fig. 1), without immune complexes.

In the absence of any suitable clinical signs or laboratory values, the acute renal failure and the glomerular changes could not be related to a known autoimmune disorder. Antiphospholipid antibody syndrome could not be taken into consideration because of a normal active thromboplastin time and absence of arteriolar and venous glomerular thrombosis on renal biopsy. Upper airway examination was completely normal and there was no sign of necrotizing granulomatous inflammation on high-resolution computerized tomography of the thorax. Thus, in the absence of characteristic clinical and serologic findings, a diagnosis of Wegener's granulomatosis was excluded. A biopsy was not performed to exclude this diagnosis.

Pulse prednisolone, $1 \mathrm{~g}$, was administered intravenously (IV), followed by cyclophosphamide, $500 \mathrm{mg}$ IV. The prednisolone was continued at a dose of $1 \mathrm{mg} / \mathrm{kg}$ per day per os. Hematemesis was produced on the second day of this treatment, and endoscopic examination showed a gastric ulcer. Examination of the endoscopic gastric biopsy specimen revealed a carcinoma of the stomach. Physical examination and imaging studies carried out after the patient's admission were negative for distant metastasis.

The patient's renal function improved with hemodialysis and immunosupressive treatment, and the patient was then transferred to the Department of Surgery, where a total gastrectomy was performed. Histopathologic examination revealed an intestinal-type adenocarcinoma of the stomach, staged as pT3N0. The specimen was negative for carcinoembryonic antigen (CEA) on immunohistologic staining, and the same was true for the initial biopsy specimen (Fig. 2). No adjuvant chemotherapy was administered for his cancer, and the steroid treatment was stopped 4 months after the operation, with his renal function being normal during all of this period. The patient has been followed regularly for 36 months, and his renal function is still normal and he is disease-free for the cancer.

\section{Discussion}

Here we have reported a patient having a gastric cancer and RPGN simultaneously. He was admitted to the hospital because of acute renal failure; treatment of glomerulonephritis was complicated by upper gastrointestinal bleeding, and a gastric cancer was found to be the cause of the bleeding. The patient improved with hemodialysis and corticosteroid treatment, and a total gastrectomy was performed. In the absence of any treatment, he has been well for approximately 3 years since the operation. 


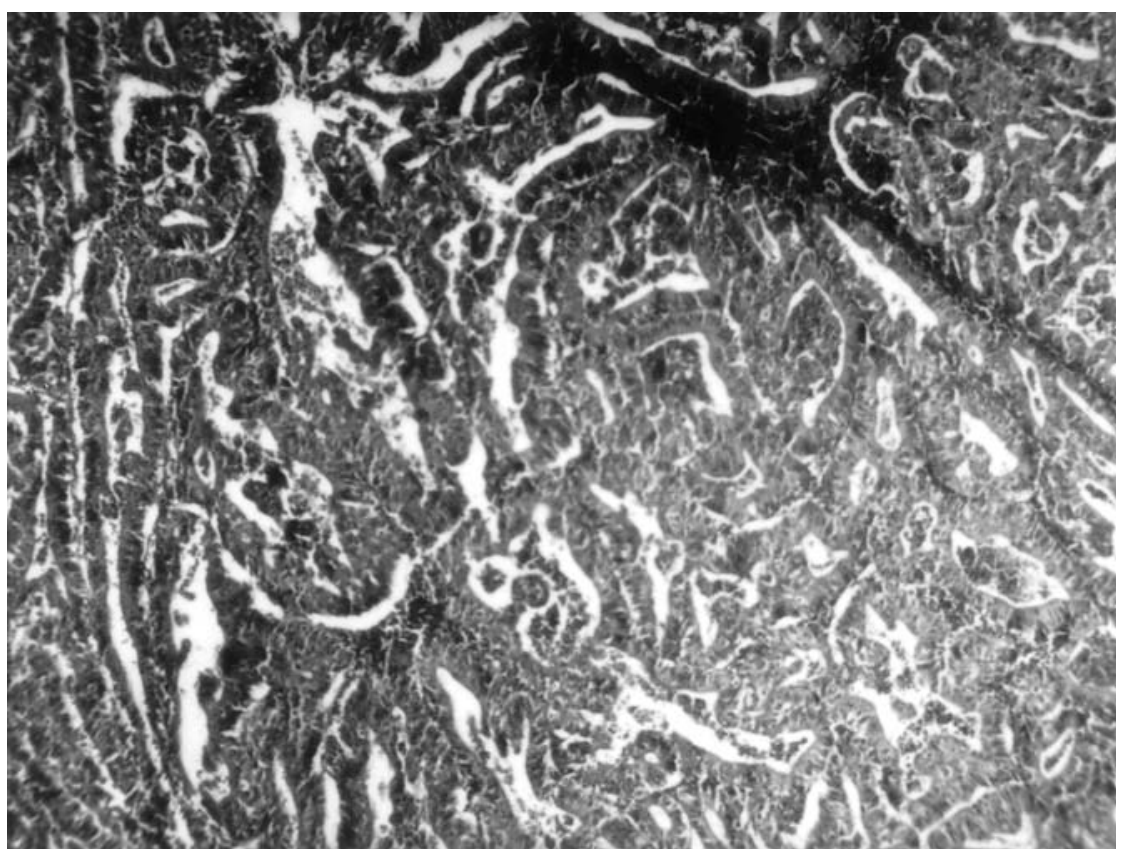

Fig. 2. Intestinal-type moderately differentiated carcinoma of stomach. $\mathrm{H} \& \mathrm{E}, \times 4$
RPGN is an uncommon syndrome that is characterized, pathologically, by focal and segmental necrosis and epithelial cell proliferation (crescents) in most glomeruli and, clinically, by fulminant renal failure associated with proteinuria, hematuria, and RBC casts. It is associated with postinfectious causes, collagen vascular diseases, immune complex diseases, autoimmune diseases, systemic necrotizing arteritis, and Wegener's granulomatosis, while, in some cases, it is idiopathic. The onset is sudden and the prognosis is poor; most patients progress to irreversible anuria in 6 months [6,7].

Malignant diseases are rare causes of glomerular pathologies such as RPGN. Immunohistochemical studies have shown complement and immunoglobulin deposition in the glomerular lesions of patients with MGN and malignancy [8]. Several tumor antigens, such as CEA, have been suggested to form antigen-antibody complexes, which are deposited on the GBM [9]. These complexes cause glomerular damage. However, tumorspecific antigen-antibody complexes are difficult to identify. The possible antigens include fetal antigens, autologous nontumor antigens, tumor-associated antigens, and viral antigens [2,7]. Removal of the tumor is sometimes associated with a remission of the acute renal failure, which may resume along with a recurrence of the tumor [2,8]. In our patient, renal function improved after steroid treatment and hemodialysis and this improvement persisted after the removal of the tumor.

In the absence of another evident cause of the RPGN and the persistent remission following the removal of the tumor, the renal disorder in our patient was considered as possible paraneoplastic nephritis. In our patient, the RPGN was the first diagnosis, and the cancer was found partly by chance because of a complication of the treatment. Thus, we can conclude that, in a patient with an acute nephritic syndrome without evident etiology, a malignant tumor could be kept in mind as an underlying disease.

\section{References}

1. Kaplan BS, Klassen J, Gault MH. Glomerular injury in patients with neoplasia. Annu Rev Med 1976;27:117-25.

2. Eagen JW, Lewis EJ. Glomerulopathies of neoplasia. Kidney Int 1977;11:297-303.

3. Zimerman SW, Vishnu-Moorthy A, Burkholder PM, Jenkins PG. Glomerulopathies associated with neoplastic disease. In: Rieselbach RE, Garnick NB editors. Cancer in the kidney. 1st Ed. Philadelphia: Lea and Febiger; 1982.

4. Stuart K, Faloon BG, Cardi MA. Development of nephrotic syndrome in a patient with prostatic carcinoma. Am J Med 1986; 80:295-8.

5. Lee J, Yamauchi H, Hopper J. The association of cancer and nephrotic syndrome. Ann Intern Med 1966;64:41-51.

6. US Renal Data System; USRDS 1997. Annual Data Report. Minneapolis: National Institute of Diabetes Digestive and Kidney Disease; 1997.

7. Dinh BL, Brassard A. Renal lesion associated with the Walker 256 adenocarcinoma in the rat. Br J Exp Pathol 1968;49:14551.

8. Alpers CE, Cotran RS. Neoplasia and glomerular injury. Kidney Int 1986;30:465-73.

9. Wagrowska-Danilewicz M, Danilewicz M. Glomerulonephritis associated with malignant diseases of nonrenal origin: a report of three cases and review of literature. Pol J Pathol 1995;46:1958 . 